\title{
FREIDLIN-WENTZELL TYPE ESTIMATES AND THE LAW OF THE ITERATED LOGARITHM FOR A CLASS OF STOCHASTIC PROCESSES RELATED TO SYMMETRIC STATISTICS
}

By

\author{
TOSHIO MORI and HIROSHI OODAIRA*
}

(Received September 22, 1988)

\begin{abstract}
Analogues of Freidlin and Wentzell's estimates for diffusion processes and the functional law of the iterated logarithm are obtained for a class of stochastic processes represented by multiple Wiener integrals with respect to two parameter Wiener processes, which arise as the limit processes of sequences of normalized symmetric statistics.
\end{abstract}

1. Introduction and results. Let $h=h\left(u_{1}, \cdots, u_{m}\right)$ be a square integrable symmetric function on $[0,1]^{m}$ and assume that $h$ is canonical, i.e., it satisfies the condition

$$
\int_{0}^{1} h\left(u_{1}, u_{2}, \cdots, u_{m}\right) d u_{1}=0 \text { for all } u_{2}, \cdots, u_{m} \in[0,1]
$$

Let $\left\{X_{j}\right\}$ be a sequence of independent identically distributed random variables uniformly distributed over $[0,1]$. Consider the following random sequence of normalized symmetric statistics

$$
Y_{n}(t)=n^{-m / 2} \sum_{1 \leq i_{1}<\cdots<i_{m} \leq[n t]} h\left(X_{i_{1}}, \cdots, X_{i_{m}}\right), \quad 0 \leqq t \leqq 1,
$$

in $D[0,1]$, the space of right continuous functions on $[0,1]$ having left limits with Skorohod's $J_{1}$ topology. A. Mandelbaum and M. S. Taqqu [3] showed that the random sequence $\left\{Y_{n}(t)\right\}$ converges weakly in $D[0,1]$ to the following process $X$ :

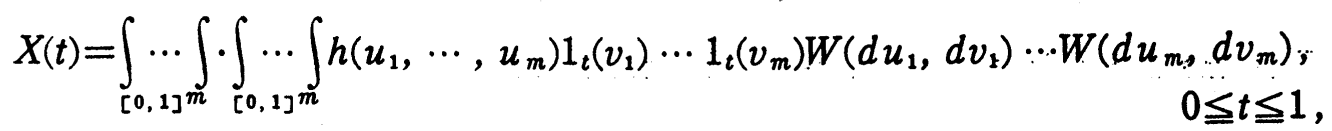

where the right hand side is an $m$-ple Wiener integral with respect to a two parameter Wiener process $\{W(u, v), 0 \leqq u, v \leqq 1\}$ and $1_{t}(\cdot)$ is the indicator func-

* Research supported by Air Force Office of Scientific Research Grant No. F49620 85C 0144 . 
tion of $[0, t] . \quad X$ has continuous paths a.s. and note also that it can be written as

$$
X(t)=\int_{[0,1]} \cdots \int_{m} h\left(u_{1}, \cdots, u_{m}\right) W_{t}\left(d u_{1}\right) \cdots W_{t}\left(d u_{m}\right), \quad 0 \leqq t \leqq 1,
$$

with $W_{t}(u)=W(u, t)$.

The purpose of this note is firstly to prove certain large deviations results, i. e., asymptotic estimates of Freidlin-Wentzell type, for the above process $X$, and secondly to remark that the functional law of the iterated logarithm for $X$ can be derived by the same arguments.

Let $C_{N}=C\left([0,1] ; R^{N}\right)$ be the space of $R^{N}$-valued continuous functions $x$ on $[0,1]$ vanishing at the origin, with the norm $\|x\|_{C}=\sup _{0 \leq t \leq 1}|x(t)|$ and the metric $d(\cdot, \cdot)$, where $|\cdot|$ stands for the Euclidean norm in $R^{N}$. Let $B=B(t)=\left(B_{i}(t)\right.$, $1 \leqq i \leqq N), 0 \leqq t \leqq 1$, be an $N$-dimensional standard Brownian motion with $B(0)=0$, and let $H_{N}$ denote the reproducing kernel Hilbert space (RKHS) associated with $B$, i. e., the Hilbert space consisting of absolutely continuous function $\varphi$ on $[0,1]$ such that $\varphi(0)=0$ and its derivative $\dot{\varphi}$ is square integrable. Its norm $\|\varphi\|_{H}$ is given by $\|\varphi\|_{H}=\|\dot{\varphi}\|_{2}=\left(\int_{0}^{1}|\dot{\varphi}|^{2}\right)^{1 / 2} \cdot H_{N}$ is a subspace of $C_{N}$ and the sets $K_{r}=$ $\left\{\varphi \in H_{N}:\|\varphi\|_{H} \leqq r\right\}, r>0$, are compact in $C_{N}$.

Define a mapping $A$ from $L^{2}[0,1] \otimes H_{1}$ (the tensor product of $L^{2}[0,1]$ and RKHS $H_{1}$ ) to $C_{1}$ by

$$
A f(t)=\int_{[0,1] m} \cdots \int_{m} h\left(u_{1}, \cdots, u_{m}\right) f\left(u_{1}, t\right) \cdots f\left(u_{m}, t\right) d u_{1} \cdots d u_{m}, \quad 0 \leqq t \leqq 1
$$

for $f \in L^{2}[0,1] \otimes H_{1}$, and let $G$ denote the class of functions

$$
G=\left\{g=A f, f \in L^{2}[0,1] \otimes H\right\} .
$$

Let $\|\cdot\|$ be the norm of $L^{2}[0,1] \otimes H_{1}$, which is given, e.g., by $\|f\|^{2}=$ $\int_{0}^{1} \int_{0}^{1}\left(\frac{\partial}{\partial t} f(u, t)\right)^{2} d u d t$. Define

and

$$
D(g)=\inf \left\{\|f\|: g=A f, f \in L^{2}[0,1] \otimes H_{1}\right\} \quad \text { for } \quad g \in G,
$$

$$
G_{r}=\{g=A f ;\|f\| \leqq r\}, \quad r>0 .
$$

The main result of this note is the following

Theorem 1. (i) For any $g \in G$ and for any $\delta, \delta^{\prime}>0$, there is a number $\alpha_{1}$ $=\alpha_{1}\left(\delta, \delta^{\prime}, D(g)\right)$ such that

$$
P\left(\|X / \alpha-g\|_{C}<\delta\right) \geqq \exp \left[-\left(\alpha^{2 / m} / 2\right)\left(D^{2}(g)+\delta^{\prime}\right)\right]
$$

for all $\alpha \geqq \alpha_{1}$, and 
(ii) for any $\delta, \delta^{\prime}, r>0$, there is a number $\alpha_{2}=\alpha_{2}\left(\delta, \delta^{\prime}, r\right)$ such that

$$
P\left(d\left(X / \alpha, G_{r}\right)>\delta\right) \leqq \exp \left[-\left(\alpha^{2 / m} / 2\right)\left(r^{2}-\delta^{\prime}\right)\right]
$$

for all $\alpha \geqq \alpha_{2}$.

In the proof of Theorem 1 we need the following Freidlin-Wentzell type estimates for $N$-dimensional Brownian motion $B$.

Theorem A (i) For any $\varphi \in H_{N}$ and for any $\delta, \delta^{\prime}>0$, there is a number $\alpha_{1}=\alpha_{1}\left(\delta, \delta^{\prime},\|\varphi\|_{H}\right)$ such that

$$
P\left(\|B / \alpha-\varphi\|_{C}<\delta\right) \geqq \exp \left[-\left(\alpha^{2} / 2\right)\left(\|\varphi\|_{H}^{2}+\delta^{\prime}\right)\right]
$$

for all $\alpha \geqq \alpha_{1}$ and

(ii) for any $\delta, \delta^{\prime}, r>0$, there is a number $\alpha_{2}=\alpha_{2}\left(\delta, \delta^{\prime}, r\right)$ such that

$$
P\left(d\left(B / \alpha, K_{r}\right)>\delta\right) \leqq \exp \left[-\left(\alpha^{2} / 2\right)\left(r^{2}-\delta^{\prime}\right)\right)
$$

for all $\alpha \geqq \alpha_{2}$.

Theorem $\mathrm{A}$ is a special case of general result on Gaussian processes, and we shall use the following easy consequences of Theorem A (see [2]).

Let $F$ be a continuous mapping from $C_{N}$ to $C_{1}$, homogeneous with degree $p>0$, i. e., satisfying the condition $F(c \cdot)=c^{p} F(\cdot)$ for any $c>0$.

Theorem B (i) For any $\varphi \in H_{N}$ and for any $\delta, \delta^{\prime}>0$,

$$
P\left(\|F(B) / \alpha-F(\varphi)\|_{C}<\delta\right) \geqq \exp \left[-\left(\alpha^{2 / p} / 2\right)\left(\|\varphi\|_{H}^{\mathscr{H}}+\delta^{\prime}\right)\right]
$$

for all sufficiently large $\alpha$, and

(ii) for any $\delta, \delta^{\prime}, r>0$,

$$
P\left(d\left(F(B) / \alpha, F\left(K_{r}\right)\right)>\delta\right) \leqq \exp \left[-\left(\alpha^{2 / p} / 2\right)\left(r^{2}-\delta^{\prime}\right)\right]
$$

for all sufficiently large $\alpha$.

\section{Theorem C}

$$
\lim _{\alpha \rightarrow \infty}\left(1 / \alpha^{2 / p}\right) \log P\left(\|F(B)\|_{C}>\alpha\right)=-b^{2} / 2
$$

where

$$
\begin{aligned}
b^{2} & =\inf \left\{\|\varphi\|_{H}^{2}:\|F(\varphi)\|_{C}>1\right\} \\
& =\sup \left\{r^{2}: \sup \left(\|F(\varphi)\|_{C}: \varphi \in K_{r}\right)<1\right\} .
\end{aligned}
$$

The arguments used in the proof of Theorem 1, combined with Strassen's law of the iterated logarithm for Brownian motion, yield also the following functional iterated logarithm law for the process $X$.

Theorem 2. Define the random sequence $\left\{Z_{n}\right\}$ in $C_{1}$ by 


$$
Z_{n}(t)=X(n t) /\left(2 n \log _{2} n\right)^{m / 2}, \quad 0 \leqq t \leqq 1, n \geqq 3,
$$

where $\log _{2}=\log \log$. Then, with probability $1,\left\{Z_{n}\right\}$ is relatively compact and the set of its limit points coincides with $G_{1}$.

Remark. The above theorem is an improvement of a recent result of $H$. Dehling [1], in the sense that a moment condition on $h$ is weakened. However, it should be noted that Dehling [1] proves more generally the functional law of the iterated logarithm for $\left\{Y_{n}(t)\right\}$. The methods of proofs are different.

\section{Proof of Theorem 1 .}

Let $\left\{e_{i}, i \geqq 0\right\}$ be a complete orthonormal sequence (CONS) in $L^{2}[0,1]$ with $e_{0}=1$. Then $\left\{e_{i_{1}}\left(u_{1}\right) \cdots e_{i_{m}}\left(u_{m}\right), i_{1}, \cdots, i_{m} \geqq 0\right\}$ is a CONS in $L^{2}\left([0,1]^{m}\right)$ and $h \in L^{2}\left([0,1]^{m}\right)$, symmetric and canonical, can be expanded as

$$
h\left(u_{1}, \cdots, u_{m}\right)=\sum_{1 \leq i_{1}, \cdots, i_{m}} c_{i_{1}, \cdots i_{m}} e_{i_{1}}\left(u_{1}\right) \cdots e_{i_{m}}\left(u_{m}\right), \quad c_{i_{1} \cdots i_{m}} \in R .
$$

For $N \geqq 1$ let

$$
h_{N}\left(u_{1}, \cdots, u_{m}\right)=\sum_{1 \leq i_{1}, \cdots, i_{m} \leq N} c_{i_{1} \cdots, i_{m}} e_{i_{1}}\left(u_{1}\right) \cdots e_{i_{m}}\left(u_{m}\right),
$$

and define the process $X_{N}=\left\{X_{N}(t), 0 \leqq t \leqq 1\right\}$ by

$$
X_{N}(t)=\int_{[0,1]^{m}} \cdots \int_{N} h_{N}\left(u_{1}, \cdots, u_{m}\right) W_{t}\left(d u_{1}\right) \cdots W_{t}\left(d u_{m}\right) .
$$

Then we have

\section{Lemma 1.}

$$
P\left(\left\|X-X_{N}\right\|_{C}>z\right) \leqq C \exp \left(-M_{N} z^{2 / m}\right)
$$

for all sufficiently large $z>0$, where $C$ is a finite constant and the positive constant $M_{N}$ can be made arbitrarily large by taking $N$ large.

Proof. The lemma follows from a result of Plikusas [5] on multiple Wiener integrals and Lemmas 6.2 and 6.3 of [4]. Indeed it is enough to note that the exponential bound obtained by Plikusas holds also for multiple Wiener integrals with respect to two parameter Wiener processes and that, putting $Z_{N}=X-X_{N}$, we have

$$
E\left|Z_{N}(t+s)-Z_{N}(t)\right|^{2} \leqq C_{N} \cdot s \quad \text { for } \quad 0 \leqq t<t+s \leqq 1 \text {, }
$$

with a finite constant $C_{N}$ and

$$
\left\|E\left|Z_{N}(t)\right|^{2}\right\|_{C}=m !\left\|h-h_{N}\right\|_{2}^{2},
$$

where $\|\cdot\|_{2}$ is the norm of $L^{2}\left([0,1]^{m}\right)$. 
Now

$$
X_{N}(t)=\sum_{1 \leq i, \cdots, i_{m} \leq N} c_{i_{1} \cdots i_{m}} \int_{[0,1] m} \cdots \int_{i_{1}} e_{1}\left(u_{1}\right) \cdots e_{i_{m}}\left(u_{m}\right) W_{t}\left(d u_{1}\right) \cdots W_{t}\left(d u_{m}\right)
$$

and, since $\left\{e_{i}\right\}$ is orthonormal, each term of the right hand side can be written as a product of Hermite polynomials, i. e.,

$$
\begin{aligned}
& \int_{[0,1]} \cdots \int_{m} e_{i_{1}}\left(u_{1}\right) \cdots e_{i_{m}}\left(u_{m}\right) W_{t}\left(d u_{1}\right) \cdots W_{t}\left(d u_{m}\right) \\
& \quad=H_{p_{1}}\left(\int_{0}^{1} e_{q_{1}}(u) W_{t}(d u)\right) \cdots H_{p_{r}}\left(\int_{0}^{1} e_{q_{r}}(u) W_{t}(d u)\right),
\end{aligned}
$$

if there are $p_{1} e_{q_{1}}(\cdot), \cdots, p_{r} e_{q_{r}}(\cdot)$ among $e_{i_{1}}(\cdot), \cdots, e_{i_{m}}(\cdot)$ with $p_{1}+\cdots+p_{r}=m$, $0<p_{1}, \cdots, p_{r}$, where $H_{p}(\cdot)$ is the $p$-th Hermite polynomial with leading coefficient 1 . Note also that

$$
B=\left(\int_{0}^{1} e_{1}(u) W_{t}(d u), \cdots, \int_{0}^{1} e_{N}(u) W_{t}(d u)\right)
$$

is an $N$-dimensional Brownian motion.

Define a mapping $T_{\dot{N}}$ from $C_{N}$ to $C_{1}$ by

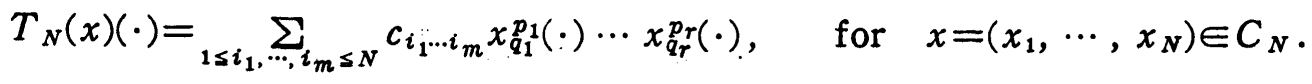

Then

$$
\begin{aligned}
X_{N}(t)-T_{N}(B)(t)= & \sum_{1 \leq i_{1} \cdots, i_{m} \leq N} c_{i_{1} \cdots i}\left\{H_{p_{1}}\left(\int_{0}^{1} e_{q_{1}}(u) W_{t}(d u)\right) \cdots H_{p_{r}}\left(\int_{0}^{1} e_{q_{r}}(u) W_{t}(d u)\right)\right. \\
& \left.-\left(\int_{0}^{1} e_{q_{1}}(u) W_{t}(d u)\right)^{p_{1}} \cdots\left(\int_{0}^{1} e_{q_{r}}(u) W_{t}(d u)\right)^{p_{r}}\right\},
\end{aligned}
$$

i. e., $X_{N}-T_{N}(B)$ is a finite linear combination of polynomials of degree $\leqq m-2$. Applying Theorem $\mathrm{C}$ to each term, we obtain

Lemma 2. For any $\delta>0$ we have

$$
P\left((1 / \alpha)\left\|X_{N}-T_{N}(B)\right\|_{C}>\delta\right) \leqq C^{\prime} \cdot \exp \left[-C^{\prime \prime} \alpha^{2 /(m-2)}\right]
$$

for sufficiently large $\alpha$, where $C^{\prime}$ and $C^{\prime \prime}$ are finite constants.

Since $T_{N}$ is clearly continuous and homogeneous with degree $m$, we get from Theorem $B$

Lemma 3. (i) For any $\varphi \in H_{N}$ and for any $\delta, \delta^{\prime}>0$,

$$
P\left(\left\|T_{N}(B) / \alpha-T_{N}(\varphi)\right\|_{C}<\delta\right) \geqq \exp \left[-\left(\alpha^{2 / m} / 2\right)\left(\|\varphi\|_{H}^{2}+\delta^{\prime}\right)\right]
$$

for sufficiently large $\alpha$, and 
(ii) for any $\delta, \delta^{\prime}, r>0$,

$$
P\left(d\left(T_{N}(B) / \alpha, T_{N}\left(K_{r}\right)\right)>\delta\right) \leqq \exp \left[-\left(\alpha^{2 / m} / 2\right)\left(r^{2}-\delta^{\prime}\right)\right]
$$

for sufficiently large $\alpha$.

Let $\left\{\phi_{j}, j \geqq 0\right\}$ be a CONS in $H_{1}$. Then $\left\{e_{i} \psi_{j}, i, j \geqq 0\right\}$ is a CONS in $L^{2}[0,1]$ $\otimes H_{1}$, and any $f \in L^{2}[0,1] \otimes H_{1}$ can be expanded as $f(u, t)=\sum_{i, j=0}^{\infty} c_{i j} e_{i}(u) \phi_{j}(t)$ with $\sum_{i, j=0}^{\infty}\left|c_{i j}\right|^{2}<\infty$. Put $\varphi_{i}(t)=\sum_{j=0}^{\infty} c_{i j} \psi_{j}(t)$. Then $\varphi_{i} \in H_{1}$ for all $i \geqq 0$,

$$
f(u, t)=\sum_{i=0}^{\infty} e_{i}(u) \varphi_{i}(t) \text { and }\|f\|^{2}=\sum_{i=0}^{\infty}\left\|e_{i}\right\|_{2}^{2}\left\|\varphi_{i}\right\|_{H}^{2}=\sum_{i=0}^{\infty}\left\|\varphi_{i}\right\|_{H}^{2} .
$$

Now, let $g=A f \in G$ with $f \in L^{2}[0,1] \otimes H_{1}$ and define

$$
g_{N}(t)=\int_{[0,1]} \ldots \int_{m} h_{N}\left(u_{1}, \cdots, u_{m}\right) f\left(u_{1}, t\right) \cdots f\left(u_{m}, t\right) d u_{1} \cdots d u_{m} .
$$

By Schwarz's inequality,

$$
\begin{aligned}
\left|g(t)-g_{N}(t)\right| & \leqq\left\|h-h_{N}\right\|_{2} \cdot\left(\int_{0}^{1} f^{2}(u, t) d u\right)^{m / 2} \\
& =\left\|h-h_{N}\right\|_{2} \cdot\left(\sum_{i=0}^{\infty} \varphi_{i}(t)\right)^{m / 2}
\end{aligned}
$$

and so

$$
\begin{aligned}
\left\|g-g_{N}\right\|_{C} & \leqq\left\|h-h_{N}\right\|_{2} \cdot\left(\sum_{i=0}^{\infty}\left\|\varphi_{i}\right\|_{C}^{2}\right)^{m / 2} \\
& \leqq\left\|h-h_{N}\right\|_{2} \cdot\left(\sum_{i=0}^{\infty}\left\|\varphi_{i}\right\|_{H}^{2}\right)^{m / 2} \\
& =\left\|h-h_{N}\right\|_{2} \cdot\|f\|^{m}
\end{aligned}
$$

Note that

$$
\begin{aligned}
g_{N}(t) & =\sum_{1 \leq i_{1}, \cdots, i_{m} \leq N} c_{i_{1} \cdots i_{m}} \int_{[0,1]} \cdots \int_{m} e_{i_{1}}\left(u_{1}\right) \cdots e_{i_{m}}\left(u_{m}\right) \cdot f\left(u_{1}, t\right) \cdots f\left(u_{m}, t\right) d u_{1} \cdots d u_{m} \\
& =\sum_{1 \leq i_{1}, \cdots, i_{m} \leq N} c_{i_{1} \cdots i_{m}}\left(\int_{0}^{1} e_{i_{1}}(u) f(u, t) d u\right) \cdots\left(\int_{0}^{1} e_{i_{m}}(u) f(u, t) d u\right) \\
& =\sum_{1 \leq i_{1}, \cdots, i_{m} \leq N} c_{i_{1} \cdots i_{m}} \varphi_{i_{1}}(t) \cdots \varphi_{i_{m}}(t) \\
& =\sum_{1 \leq i_{1}, \cdots, i_{m} \leq N} c_{i_{1} \cdots i_{m}}\left(\varphi_{q_{1}}(t)\right)^{p_{1} \cdots\left(\varphi_{q_{r}}(t)\right)^{p_{r}}} \\
& =T_{N}(\varphi)(t), \quad \text { where } \varphi=\left(\varphi_{1}, \cdots, \varphi_{N}\right) \in H_{N},
\end{aligned}
$$

and also that, for any given $\varphi=\left(\varphi_{1}, \cdots, \varphi_{N}\right) \in H_{N}$, if we put $f(u, t)=\sum_{i=1}^{N} e_{i}(u) \varphi_{i}(t)$, then $f \in L^{2}[0,1] \otimes H_{1},\|f\|=\|\varphi\|_{H}$ and $A f=T_{N}(\varphi)$. 
From the above we immediately obtain the following

Lemma 4 (i) For any $g=A f \in G$ with $f \in L^{2}[0,1] \otimes H_{1}$ and for any $\delta>0$, there is an element $g_{N}$ of the form $g_{N}=T_{N}(\varphi)$ with $\varphi \in H_{N},\|\varphi\|_{H} \leqq\|f\|$ such that $\left\|g-g_{N}\right\|_{C}<\delta$ for all sufficiently large $N$.

(ii) $T_{N}\left(K_{r}\right) \subset G_{r}$ for all $r>0, N \geqq 1$.

Proof of Theorem 1 (i) Let $g \in G, \delta, \delta^{\prime}>0$ be given, and assume that $g$ is of the form $g=A f, f \in L^{2}[0,1] \otimes H_{1}$. Choose $N$ large enough that $\left\|g-g_{N}\right\|_{C}$ $<\delta / 4$ with $g_{N}=T_{N}(\varphi), \varphi \in H_{N}(\operatorname{Lemma} 4(\mathrm{i}))$, and $M_{N}>(4 / \delta)^{2 / m}\left(\|f\|^{2}+\delta^{\prime}\right)$ (cf. Lemma 1). Then, by Lemmas 1,2 and $3(\mathrm{i})$,

$$
\begin{aligned}
P\left(\|X / \alpha-g\|_{C}<\delta\right) \geqq & P\left(\left\|T_{N}(B) / \alpha-T_{N}(\varphi)\right\|_{C}<\delta / 4\right) \\
& -P\left(\left\|X-X_{N}\right\|_{C}>\alpha \delta / 4\right) \\
& -P\left(\left\|X_{N}-T_{N}(B)\right\|_{C}>\alpha \delta / 4\right) \\
\geqq & \exp \left[-\left(\alpha^{2 / m} / 2\right)\left(\|\varphi\|_{H}^{2}+\delta^{\prime} / 3\right)\right] \\
& -C \cdot \exp \left[-M_{N}(\alpha \delta / 4)^{2 / m}\right] \\
& -C^{\prime} \cdot \exp \left[-C^{\prime \prime} \alpha^{2 /(m-2)}\right]
\end{aligned}
$$

for all sufficiently large $\alpha$. Noting that $\|\varphi\|_{H} \leqq\|f\|$, we thus obtain

$$
P\left(\|X / \alpha-g\|_{C}<\delta\right) \geqq \exp \left[-\left(\alpha^{2 / m} / 2\right)\left(\|f\|^{2}+2 \delta^{\prime} / 3\right)\right]
$$

for all sufficiently large $\alpha$, and the assertion follows from the definition of $D(g)$.

Proof of Theorem 1 (ii) Given $\delta, \delta^{\prime}, r>0$, choose $N$ large enough that $M_{N}>(3 / \delta)^{2 / m}\left(r^{2}-\delta^{\prime}\right)$ (cf. Lemma 1). Then, by Lemmas $1,2,3$ (ii) and 4(ii),

$$
\begin{aligned}
P\left(d\left(X / \alpha, G_{r}\right)>\delta\right) \leqq & P\left(d\left(X / \alpha, T_{N}\left(K_{r}\right)\right)>\delta\right) \\
\leqq & P\left(d\left(T_{N}(B) / \alpha, T_{N}\left(K_{r}\right)\right)>\delta / 3\right) \\
& +P\left(\left\|X-X_{N}\right\|_{C}>\alpha \delta / 3\right)+P\left(\left\|X_{N}-T_{N}(B)\right\|_{C}>\alpha \delta / 3\right) \\
\leqq & \exp \left[-\left(\alpha^{2 / m} / 2\right)\left(r^{2}-\delta^{\prime} / 2\right)\right] \\
& +C \cdot \exp \left[-M_{n}(\alpha \delta / 3)^{2 / m}\right]+C^{\prime} \cdot \exp \left[-C^{\prime \prime} \alpha^{2 /(m-2)}\right] \\
\leqq & \exp \left[-\left(\alpha^{2 / m} / 2\right)\left(r^{2}-\delta^{\prime}\right)\right]
\end{aligned}
$$

for all sufficiently large $\alpha$. This completes the proof of Theorem 1 .

\section{Proof of Theorem 2 .}

It follows from Lemmas 1 and 2 that

$$
P\left(\left\|X-T_{N}(B)\right\|_{C}>z\right) \leqq \exp \left[-M z^{2 / m}\right]
$$


for all sufficiently large $z$ and $N$, where $M$ is any given positive constant. Note also that both processes $X(\cdot)$ and $T_{N}(B)(\cdot)$ are self-similar with parameter $m / 2$, i.e., $X(c \cdot)$ and $c^{m / 2} X(\cdot)$, and also $T_{N}(B)(c \cdot)$ and $c^{m / 2} T_{N}(B)(\cdot)$, have the same finite dimensional distributions for any $c>0$. Using these facts and the first Borel-Cantelli lemma, we get by the standard argument

Lemma 5. For any $\varepsilon>0$, with probability 1

$$
\limsup _{n \rightarrow \infty}\left\|Z_{N}-T_{N}(B)(n t) /\left(2 n \log _{2} n\right)^{m / 2}\right\|_{C} \leqq \varepsilon
$$

for sufficiently large $N$.

Note that

$$
T_{N}(B)(n t) /\left(2 n \log _{2} n\right)^{m / 2}=T_{N}\left(B_{n}\right)(t),
$$

where $B_{n}(t)=B(n t) /\left(2 n \log _{2} n\right)^{1 / 2}$. Thus, by Strassen's law of the iterated logarithm for $B$ and the continuous mapping theorem, we have

Lemma 6. For any $N \geqq 1$, with probability 1 the random sequence $\left\{T_{N}\left(B_{N}\right)\right.$, $n \geqq 3\}$ in $C_{1}$ is relatively compact and the set of its limit points is $T_{N}\left(K_{1}\right)=$ $\left\{T_{N}(\varphi) ; \varphi \in K_{1}\right\}$.

Theorem 2 follows from Lemmas 4, 5 and 6.

\section{References}

[1] Dehling, H.: The functional law of the iterated logarithm for von-Mises-functionals and multiple Wiener integrals. Preprint, 1984 (revised 1987).

[2] Kallianpur, G. and Oodaira, H.: Freidlin-Wentzell type estimates for abstract Wiener spaces. Sankhya, Ser. A, 40 (1978), 116-137.

[3] Mandelbaum, A. and Taqqu, M.S.: Invariance principle for symmetric statistics. Ann. Statistics, 12 (1984), 483-496.

[4] Mori, T. and Oodaira, H.: The law of the iterated logarithm for self-similar processes represented by multiple Wiener integrals. Probab. Th. Rel. Fields, 71 (1986), 367-391.

[5] Plikusas, A.: Some properties of the multiple Ito integral (in Russian). Liet. Mat. Rink., 21 (1981), 163-173.

Department of Mathematics

Yokohama City University

22-2 Seto, Kanazawa-ku,

Yokohama 236 Japan

Department of Applied Mathematics

Yokohama National University

156 Tokiwadai, Hodogaya-ku

Yokohama 240 Japan 